In dieser (primär) rechtswissenschaftlich ausgerichteten Arbeit finden Landwirtschaftsrechtler vielfaches Material zu rechtsvergleichenden Studien. Als Beispiel sei hier auf das Fehlen eines besonderen landwirtschaftlichen Erbrechts verwiesen, wie es die Bundesrepublik mit ihren Bestimmungen über das Anerben- und Zuweisungsrecht kennt. Aus der Anwendung der Grundsätze des japanischen BGB (gleichmäßige Aufteilung des Nachlasses unter allen Erben, wobei die Rechtstellung der Frau sehr schwach ist) wird der Erhalt landwirtschaftlicher Betriebe gefährdet und die Realteilung landwirtschaftlichen Grundeigentums begünstigt.

Darüber hinaus ist die Arbeit aber auch interessant für (Rechts-)Soziologen und Politikwissenschaftler. Sie geht an einigen Stellen über den bloß rechtswissenschaftlichen Bereich hinaus und stellt (wenn auch nur knapp) Verknüpfungen zwischen den Gesetzen und den ökonomischen und soziopolitischen Bedingungen ihrer Entstehung her. So lernen wir, daß die landwirtschaftliche Bodenreform Japan nicht ganz von den USA aufgezwungen, sondern auch aus eigenem Antrieb mit Unterstützung der amerikanischen Okkupationsmacht durchgeführt wurde. Dabei konnte die eine Reform der Pachtverhältnisse behindernde Gruppe von ,,absentee landlords“, von Tayama treffend ,,schmarotzende Grundbesitzer“" genannt, ausgeschaltet werden.

Durch die von 1947 bis 1949 durchgeführte umfangreiche Bodenreform konnte der Anteil von Pachtland an allen bewirtschafteten Flächen von $46 \%$ auf $10 \%$ gesenkt werden. Von den etwa 2,3 Mio. ha Pachtland wurden durch Zwangsankauf etwa $80 \%$ auf selbstwirtschaftende Landwirte übertragen. Landwirtschaftliches Großgrundeigentum wurde beseitigt. Nur im Dorf ansässige Grundbesitzer durften noch 1 ha (in Hokkaido etwa 4 ha) behalten. Diese radikale Veränderung der Landverfassung, begleitet von einer drastischen Senkung der Pachtzinsen, schuf zusammen mit der Demokratisierung der wirtschaftlichen, gesellschaftlichen und politischen Strukturen die Voraussetzung für den Aufschwung Japans zur Wirtschaftsmacht heutiger Größe.

Auch wenn diese Entwicklung nicht nachholbar sein dürfte und aus soziopolitischer Sicht nicht nur positive Ergebnisse gebracht hat, so kann der Weg Japans mit einer radikalen Änderung der Landbesitzstrukturen auf breiter Basis als Voraussetzung für wirtschaftliche und soziale Entwicklung für viele landwirtschaftlich strukturierte Gesellschaften Südostasiens, in denen das Problem Landreform ansteht, richtungweisend sein für die Implementierung eines Reformprogramms per Gesetz von oben herab.

Ulrich Rausch

KenNETH F. Johnson

Mexican Democracy: A Critical View

Rev. Edition, New York, London, Sydney, Toronto, 1978, 267 S.

Peter H. Smith

\title{
Labyrinths of Power
}

Princeton, New Jersey, 1979, XVI, 384 S.

Angesichts der seit Jahrzehnten bestehenden Dominanz des Partido Revolucionario Institucional - PRI - im politischen Leben Mexikos stellt die Wahl des von ihm aufgestellten Präsidenten kaum mehr als einen Akklamationsprozeß dar, so daß entscheidend nur die parteiinterne Kandidatenaufstellung ist. Das Lancieren der geeignet erscheinenden Anwärter auf die Kandidatur ist letztlich in seinen Vorbereitungen, Absprachen, Rücksichtnahmen nicht prinzipiell verschieden von den ,Findungsverfahren“ in anderen politischen Systemen. 
Mögen diese noch so ,,offen“ und demokratisch sein, so setzt doch jeder Beginn eines Abstimmungsverfahrens zwangsläufig voraus, daß die jeweiligen Gruppierungen ,,ihren“ Anwärter und diesen wiederum mit entsprechendem Rückhalt präsentieren müssen. Diese internen Auswahlverfahren im Vorfeld in ihrer für den Außenstehenden Undurchsichtigkeit sind beliebter Gegenstand unzähliger Arbeiten, um diese, offenbar als undemokratisch angesehenen Manipulationen ,,aufzudecken" und doch noch in eine, dem Eingeweihten und Experten erschließbaren ,,Gesetzmäßigkeit“ einzubauen. Dies ist z. T. auch der Ansatz von Johnson, der in dem PRI keine Partei, sondern einen die undemokratischen Praktiken des Präsidenten deckenden Interessenklüngel ${ }^{1} \mathrm{zu}^{\mathrm{z}}$ sehen vermag. Seine interessante, faktenreiche Arbeit ${ }^{2}$ stellt sich streckenweise geradezu als Abrechnung mit der Echeverría-Zeit dar. Diese zeit- und personenbezogene chronique scandaleux läßt schwerlich anerkannte Grundlinien der politischen Entwicklung anhand der besprochenen Präsidentenwechsel sichtbar werden. Entscheidend ist nämlich nicht das operative Wie, sondern das substantielle Warum der konkreten Kandidatur.

Smith arbeitet dazu die soziale Herkunft des in Frage kommenden Führungskräftereservoirs des PRI auf mit ausführlicher Katalogisierung der für Herrschaft in Mexiko insgesamt und für den PRI speziell notwendigen Elemente. Das Ergebnis, belegt mit Untersuchungen zeitlich bis zur Jahrhundertwende zurückreichend, überrascht sicherlich niemanden: Die politische Elite wird von der oberen Mittelklasse gestellt. Smith liefert hierzu ergänzende Subklassifizierungen etwa im Hinblick auf die regionale Herkunft der Angehörigen der oberen Klassen (S. 66-71): Während unter Díaz Anfang des Jahrhunderts die zentralen Bundesstaaten einschließlich des Distrito Federal zahlenmäßig deutlich unterrepräsentiert waren und sich dies während der Revolutionszeiten kaum besserte - statt dessen drangen die Repräsentanten des Nordwestens, die Soronenser vor, - konnten sich in der nachrevolutionären Zeit (1946-1971) die Angehörigen aus den Zentralstaaten (capitalinos) entsprechend dem Bevölkerungsanteil durchsetzen, darüber hinaus waren jetzt die Vertreter der Südstaaten mit zusammen 31,1 \% bei einem Bevölkerungsanteil von nur 19,5\% in der Elite vertreten. Wichtiger ist indes die Herkunft aus dem ländlichen oder urbanen Bereich sowie die bildungsmäßige und berufliche Einbindung. Das Ergebnis seit den sechziger Jahren ist eindeutig: Die politische Elite ${ }^{3}$ ist städtisch ${ }^{4}$ mit Hochschulbildung, Rechtsanwälte, Lehrer und Hochschullehrer überwiegen. Militärs - vorrangig von ländlicher Herkunft und geringer Schulbildung (Smith, S. 95/6) ${ }^{\mathbf{5}}$, naturgemäß in den Revolutionszeiten mit wenigstens einem Drittel in der Elite vertreten, haben heute kaum mehr eine Bedeutung. Zentrum der hochschulmäßigen Ausbildung künftiger Politiker ist im übrigen nachweisbar die Universidad Nacional Autónoma de México (UNAM) in der Hauptstadt.

Die Regeln, nach denen Positionen in den oberen Etagen des mexikanischen Regierungssystems erreicht werden können (Smith, S. 242 ff.), sind im Kern sicherlich auch auf andere Systeme anwendbar: Einflußreiche Freunde vor allem bei seinen Vorgesetzten gewinnen, die einen fördern können. Familiäre Verbindungen, auch Schwägerschaften, schaden nicht (Sy-

1 S. 62.

2 Auszugsweise auch bei DiBacco (Hrg.), Presidential Power in Latin American Politics, New York-London, 1977, S. 33-55, abgedruckt.

3 Von Smith im Anhang A -S. 317 ff. - in Anlehnung an Frank Tannenbaum, The Making of Modern Mexico, 1964, S. 158 ff., gegliedert vom Präsidenten über Mitglieder des Kabinetts, Parteispitzen, Gouverneure, Senatoren bis hin zu den Bundesdeputierten und den Inhabern wichtiger Botschafterposten, u. a. in Deutschland oder Frankreich. Der Präsident der Suprema Corte wird bei Smith nicht erwähınt, bei Brandenburg in den Rang der Senatoren und Staatssekretäre eingeordnet. So ist der vormalige Gerichtspräsident Guerrero López 1976 zum Senator für Guanajuato gewählt worden.

4 Camp, Foro Internacional, Bd. XIX, Num. 1 (1978), S. 24 (26). Zum Rückgang des Militärs seit 1934 Stansfiled, The Mexican Cabinet: An indicator of political chance, Glasgow 1973, S. 3; speziell für den Bereich der Gouverneursposten und den PRI, vgl. Boils, Los militares y la política en México (1915-1974), México, 1975, S. 175 ff.

5 Zuletzt wurde 1940 mit Avila Camacho ein General Präsident. 
stem des indirekten Nepotismus). In die Gefolgschaft (equipo oder camarilla) eines aufstrebenden Politikers treten, ohne sich jedoch Feinde zu schaffen, wie man überhaupt Probleme nicht angehen und Fehler vermeiden sollte (Erklärung für Immobilismus). Das Verfassungsprinżip der no reelección mit seinem daraus folgenden 3-Jahres- bzw. 6-Jahresrhythmus (auf Deputierten- und Senatoren- bzw. Präsidentenebene, Art. 59, 83 der Verfassung) schlägt auf die internen Karriereregeln durch ${ }^{6}$ : Jeder Amtsinhaber hat damit zu rechnen, daß einer seiner jetzigen Untergebenen in einer der nächsten Amtsperioden - sei es auch in einem ganz anderen Geschäftsbereich - nunmehr selbst Vorgesetzter ist. Dies in Rechnung stellend muß jedermann seine Untergebenen hofieren ${ }^{7}$. Freundschaft und Rücksichtsnahmen haben allerdings dort ein Ende, wo die Spitze der Hierarchie erreicht wird und Bewerber nebst Anhang alles auf eine Karte setzen müssen, beim Staatspräsidentenamt. Zunächst bestehen verfassungsrechtliche und psychologische Barrieren: Nach Art. 35, 36 der Verfassung stehen die politischen Rechte und Pflichte allen mexikanischen Staatsbürgern zu. Für das Präsidentenamt jedoch muß der Amtsinhaber von Geburt an Mexikaner sein und Abkömmling gebürtiger mexikanischer Eltern, Art. 82, Ziff. I. Diese Verfassungsbestimmung ist von interessierter Seite immer wieder angegriffen worden als diskriminierend ${ }^{8}$. Mit der erwähnten Eingrenzung werden so potente Leute aus dem Bewerberkreis ausgeschlossen wie Reyes Heroles, Sohn eines Spaniers; Hank González, Sohn eines Deutschen, und Oteyza, Kind spanischer Eltern ${ }^{9}$. Weiter erscheint es auch heute noch ausgeschlossen, daß eine Frau auch nur als Bewerberin in Betracht kommt ${ }^{10}$. Wer Bewerber um die Präsidentschaftskandidatur ist, bleibt wenigstens für die nächste Amtsperiode - sexenio - vom Postenkarussell ausgeschlossen, wenn der Mitbewerber siegt. Mit dem Unterlegenen haben all diejenigen seiner camarilla verloren, die sich zu sehr öffentlich für ihren Favoriten eingesetzt haben, indes sich auch so einsetzen mußten, wollten sie bei einem Sieg führende Positionen, d. h. ein Ministeramt in der Bundesregierung oder einen Gouverneursposten erhalten. Selbst für die Vorsichtigen kommt der Augenblick, in welchem sie sich noch vor der Bekanntgabe des wahren Präsidentschaftskandidaten - verdadero tapado - entscheiden müssen, wollen sie vorwärts kommen oder einen Abstieg verhindern. Das Bekenntnis nach der Bekanntgabe - destapamiento11 - nützt wenig in der unübersehbar werdenden Schar der Jubilanten (quasi der „März-Gefallenen“).

Smith ${ }^{12}$ wie auch Johnson ${ }^{13}$ schildern mit Wiedergabe des Berichts von Cosío Villegas die Bekanntgabe des neuen Präsidentschaftskandidaten López Portillo am Ende der Amtszeit von Echeverría Alvarez. Da dies oft kolportiert worden ist, soll hier nur dem Schicksal des aussichtsreichsten Mitbewerbers Moya Palencia nachgegangen werden: Sein Vorteil gegenüber den 6 Mitbewerbern ${ }^{14}$ war der, daß er das Innenministerium unter Echeverría innehatte und damit das Amt, welches in der Vergangenheit Sprungbrett für das Präsidentenamt war:

\footnotetext{
6 Während für den Staatspräsidenten ein absolutes Wiederwahlverbot gilt, können etwa Deputierte in der übernächsten Periode wiedergewählt werden, so daß sich für einen Berufspolitiker wie Farías folgende Sequenz ergibt: Deputierter 1955-1958, 1967-1970, 1979-1982, dazwischen Senator und Gouverneur in Nuevo Leon.

7 Dies gilt auch für den Universitätsbereich, wenn dort ein Politiker als Prof essor abwartet und später von einem ehemaligen Studenten für ein Regierungsamt wiederendeckt wird, vgl. das Beispiel des vormaligen Pemex-Generaldirektors Dovali bei Smith, S. 273.

8 González, in: Novedades v. 28. 11. 1977, S. 4, unter Hinweis auf diese fehlende Einschränkung in der Verfassung von 1857. Ex-Präsident Alemán bekannte sich zur Reform des Art. 82, Novedades v. 30. 11. 1977, S. 1.

9 Und konnten daher gefahrlos in zentrale Positionen unter López P. einrücken: Reyes Heroles, ehemaliger Präsident und Chefideologe des PRI, wurde Innenminister; Hank González trat an die Spitze des D.F., und der am 21.11. 1942 geborene Oteyza übernahm als jüngstes Kabinettsmitglied das Ministerium für Bundesvermögen.

10 Von Farías (vgl. Anm. 6), Leiter der Gran Comisión der Deputiertenkammer, eingeräumt, La Prensa v. 14. 3. 1980 , S. 6.

11 Den Uberraschungseffekt für die Bevölkerung betont Cosío V., El sistema político mexicano, 7. Aufl., México, 1974, S. 93.

12 S. 287 f.

13 S. 58.

14 Neben López Portillo, dem Finanzminister: Cervantes del Río, Staatsminister im Präsidialamt; Muñoz Ledo, Arbeitsminister; Gálvez Betancourt, Generaldirektor des Instituto Mexicano del Seguro Social; Gómez Villanueva, Agrarreformminister, und schließlich Bracamontes, Minister für öffentliche Arbeiten.
} 
So war Echeverría 1958 Staatssekretär unter dem Innenminister Díaz Ordaz, und als dieser, wie früher schon Alemán und Ruiz Cortines, Präsident wurde (1964) rückte Echeverría nach als Innenminister, um 1970 wiederum Nachfolger von Díaz Ordaz zu werden ${ }^{15}$. Ähnlich schien die Karriere von Mario Moya Palencia zu verlaufen: 1969 Staatssekretär im Innenministerium und 1970 Nachfolger Echeverrías im Ministeramt. Mit dem Fehlschlagen seiner Kandidatur mußte Moya von der politischen Plattform abtreten ${ }^{\mathbf{1 6}}$, un den neuen Präsidenten nicht zu behindern. Er übernahm eine Professur an der UNAM und kann dort das Ende des Sexeniums abwarten ${ }^{17}$ 18. Warum entschied sich der innere Zirkel um Echeverría mit dem Gewerkschaftsführer Fidel Velázquez Sánchez ${ }^{19}$ für López Portillo? Jugendfreund Echeverrías hatte López in dem PRI kaum eine eigene Machtbasis. Der Versuch, mit einem daher von Unterstützung abhängigen Präsidenten indirekt, d. h. unter formaler Wahrung des Wiederwahlverbotes, weiterregieren $\mathrm{zu}$ wollen ${ }^{\mathbf{2 0}}$, die Begründung eines maximatos ${ }^{21}$, schien aufder Hand zu liegen. Die Unruhen ${ }^{22}$ vor der Amtsübergabe am 1. Dezember 1976 schienen nachdrücklich das Erfordernis einer Unterstützung durch Echeverría anzuzeigen. Der hohe Wahlsieg23 des simpatico ${ }^{24}$ López Portillo am 4. Juli 1976 hatte jedoch schon eine Grundlage für die Abkehr von der bisherigen Herrschaft angezeigt. López P. ist es wie noch

15 Echeverría hatte als bisher einziger Präsident nie ein Legislativamt inne, Smith, S. 279; Womack, Foreign Affairs, Bd. 48 No. 4 (1970), S. $677(684 / 5)$.

16 Am 25. 10. 1975 mußte ein Moya-Anhänger, der Gouverneur von Sonora, Carlos Armando Biebrich Torres, sein Amt auf Druck aus dem Präsidentenpalast aufgeben, Smith, S. 269. Näher zur Biebrich-Affäre Johnson, S. 180-183. Jetzt ist Biebrichnach Ablauf des Sexeniums von Echeverría wieder politisch tätig,Novedades v. 11. 7. 1980, S. 14. Auch anläßlich diesesFalles zeigt sich, daß die Entsetzung eines mißliebigen Gouverneurs - unter Echeverría allein in fünf Fällen - kein Beispiel einer grundsätzlichen Auseinandersetzung zwischen Bund und Einzelstaat ist, sondern schlicht die Bereinigung einer parteiinternen Querele. - Auch der von Johnson, S. 87-92, ausgebreitete Fall des Aufstiegs von Alfonso Martínez Domínguez unter Díaz Ordaz und sein Abstieg unter Echeverría hat die von Johnson, S. 92, prognostizierte Entwicklung unter dem neuen Präsidenten genommen: Martínez ist 1979 Gouverneur von Nuevo Leon geworden.

17 Ein Parallelfall dazu ist das politische Schicksal von Emilio Martínez Manautou, dem erfolglosen Mitbewerber um die Präsidentschaft 1970, der daher im Exenium Echeverrías pausieren mußte (quemado - ausgebrannt), um dann unter López P., früher Untergebener von Martínez M., Gesundheitsminister zu werden, Smith, S. 300. Am 5. 6. 1980 ist er zurückgetreten, um das (höherwertige) Gouverneursamt für Tamaulipas, seinem Heimatstaat, zu übernehmen, Comercio Exterior 1980, S. 552, d. h. augenfällig Sicherung von Gouverneursposten über die Präsidentenzeit 1982 hinaus durch einen lopezportillista zum einen. Als Unterstützung enteignete López P. It. D.O. v. 20. 5. 1980505000 ha Viehweiden in Tamaulipas für den Getreideanbau. Zum anderen dient diese Wahl der Beruhigung des Grenzstaates zu den USA (vgl. zu den Zuständen in Tamaulipas Excelsior v. 9. 1. 1978; Latin America Political Report v. 20. 1. 1978, S. 20/1). In das Ministeramt rückte dessen Staatssekretär Mario Calles López Negrete ein.

18 Die anderen Mitbewerber ohne ausreichenden Rückhalt wie Gálvez Betancourt und Bracamontes blieben deswegen unberücksichtigt. Muñoz Ledo und Cervantes del Río dagegen, Echeverría-Anhänger, wurden mit bedeutenden, jedoch auch gefährlichen Ämtern bedacht: Muñoz mußte sich als Erziehungsminister mit Studentenunruhen herumschlagen - er schied inzwischen am 9. 12. 1977 aus dem Kabinett aus -, Cervantes del Río als Direktor der CFE mit der Elektrikergewerkschaft.

19 Der 1900 geborene Führer (lider) des Gewerkschaftsbundes CTM, seit 1941 an seiner Spitze, ist bereits heute nationales Denkmal. Die Zugehörigkeit der Gewerkschaftsführer zum Machtkartell mag hier angedeutet sein, wo lediglich den parteiinternen Wegen nachgegangen wird. Der Hinweis auf die gewerkschaftlichen Verflechtungen soll Erinnerungspunkt dafür sein, daß das mexikanische Herrschaftssystem verästelter ist als es sich in einer Rezension darstellen läßt, vgl. dazu Domitra. Die Rolle der Gewerkschaften im mexikanischen Herrschaftssystem, Bonn, 1975, passim.

20 Ein gänzlich neues und unangreifbares Vehikel hierfür war die Ausarbeitung eines ,,Plan basico de gobierno“ durch den PR I für das bevorstehende Sexenium, Smith, S. 285, bereits 1975, den kein Anwärter angreifen konnte, wollte er sich nicht selbst aus dem Rennen werfen, und der ihn daher für die Zukunft band. Ein Anliegen Echeverrías war das der stärkeren Medienkontrolle: Im Plan basico wurde eine Ergänzung des Art. 6 der Verfassung (Meinungsfreiheit) um ein staatlich garantiertes Informationsrecht gefordert, vgl. dazu Burgoa, Las Garantias Individuales, 12. Aufl., México, 1979, S. 678/9, und unter López eingeführt, D.O. v. 6. 12. 1977, vgl. dazu Scheffler, in: Uberseeische Verfassungsvergleichung I, Hamburg, 1980, S. 35 (46/7).

21 Nach Ende seiner Amtszeit 1928 herrschte Präsident Calles mit ihm gefügigen Nachfolgern (Portes Gil, Ortiz Rubio und Rodríguez) und der von ihm gegründeten PRI - damals unter dem Namen Partido Nacional Revolucionario, vgl. Furtak, Revolutionspartei und politische Stabilität in México, Hamburg, 1969, S. 16 ff. - weiter. Diese Zeit wird als maximato bezeichnet. Erst Cárdenas konnte die Tutel Calles' abschütteln.

22 Anschläge auf Angehörige von Echeverría und López Portillo, Landbesetzungen im Norden des Landes, Floating des Pesos, Anwürfe gegenüber der Unternehmensgruppe in Monterrey, Warnung vor Faschismus, S. 292 ff., schienen inszeniert zu sein, um die Notstands klausel des Art. 84 der Verfassung in Kraft zu setzen, nach der ein Interimspräsident an Stelle des nicht ins Amt gelangenden designierten Präsidenten bestellt werden kann. Indes konnte jener verfassungsgemäß nie der scheidende Präsident sein, Art. 85, so daß auf diesem Wege eine Weiterherrschaft Echeverrías ohnehin nicht möglich gewesen wäre.

$2394,4 \%$ der abgegebenen Stimmen bei einer Wahlbeteiliguung von 68,8 \%, indessen dadurch begründet, daß die Opposition PAN diesmal keinen Gegenkandidaten zu stellen vermochte. Zu den parteiinternen Auseinandersetzungen vgl. Johnson, S. 145 ff. Für den PRI verlagerte sich angesichts des bloßen Akklamationsprozesses der Wahl die Stimmabgabe zu einer Frage des Legitimationsgrades, so nachdrücklich Lira M., in: línea H. 22 (1976), S. $121 \mathrm{ff}$.

24 Als Gegensatz zu Echeverría stilisiert, dem etwa am 7. 9. 1979 von der Universität Guadalajara die erst am 15. 4. 1975 verliehene Ehrendoktorwürde mit der Begründung aberkannt wurde, er sei ein ,,Verräter und Mörder“, d. h. für den Tod eines Studenten mitverantwortlich, Impacto v. 19. 9. 1979, S. 6. 
jedem Präsidenten seit Cárdenas gelungen, sich von den Bindungen seiner ehemaligen Promotoren zu lösen und sich eine eigene Machtbasis zu schaffen ${ }^{25}$. Die Zusammensetzung seines Kabinetts und die Besetzung der nachgeordneten Positionen mit persönlichen Freunden und Familienangehörigen - personale Bindungen - und Berücksichtigung von Angehörigen aller Parteigruppierungen und Anhängern früherer Präsidenten ${ }^{26}$ zeigen die Eigenständigkeit des Präsidenten und die akkurate Einhaltung der parteiinternen Spielregeln. Derartige Verhaltensweisen im Spitzenamt sind zugleich Ausdruck der Flexibilität des Systems selbst: Das Verprellen des Privatsektors durch Echeverría hatte zu einem gefährlichen Investitionsattentismus der Unternehmer geführt. Hier die Balance im gemischtwirtschaftlichen System Mexikos pragmatisch wieder austariert zu haben, ist Verdienst von López P. Sein politisches Credo war daher naheliegend: die Alianza para la producción ${ }^{27}$, mit der als erstes die privaten Investitionen wieder angekurbelt und die aufgerissenen Gegensätze eingeebnet werden sollten.

Dauerndes Problem einer immerwährenden Einparteienherrschaft ist die Integration unzufriedener politischer Kräfte ${ }^{28}$. Noch hat der PRI mit der Gewährung von Aufstiegschancen und Verkündung immer neuer, zeitangepaßter Verheißungen durch äußerlich so unterschiedliche Präsidenten die innenpolitische Szene beherrschen können. Nicht anders war es beim Úbergang von Echeverría auf López, und nicht anders wird es sein beim Präsidentenwechsel 1982. Echeverría bot die apertura democrática vornehmlich den jungen Akademikern an ${ }^{29}$ und umgab sich selbst mit einem Ring jüngerer Technokraten, der efebocracia ${ }^{\mathbf{3 0}}$. Unter López Portillo wurde das erstickende Mehrheitswahlsystem in ein additives Mehrheits- und Verhältniswahlrecht umgestaltet mit der Folge von Parteineugründungen am äußersten rechten und linken Rand der politischen Szene. Der Umstand, daß der PRI selbst diese politische Reform getragen hat, war indes Beweis genug, daß seine Herrschaft nicht ernstlich angefochten sein würde ${ }^{\mathbf{3 1}}$.

Mit dem sich nähernden Ende des Sexeniums von López Portillo beginnt eine neue Runde um die Anwartschaft auf das Präsidentenamt. Die Zeit, die Startlöcher zu graben, von denen aus die potentiellen Bewerber antreten werden, ist ohnehin da: Das Feld ist das der Wirtschaftspolitik gewesen. Die Diskussion um einen Beitritt zum GATT war im Kabinett, dem primären Kandidatenreservoir, öffentlich kontrovers ${ }^{32}$.

Die Herrschaft des PRI in Mexiko vermag aus sich selbst heraus personelle Alternativen zu produzieren und komplettiert damit das System, selbst alle Varianten einer politischen Auseinandersetzung anbieten zu können: Der PRI ist etwa im Bereich der Agrarreformen selbst Opfer und Täter bei Landbesetzungen, Ankläger und Untersuchungsführer für Übergriffe, Richter und Gnadeninstanz für getroffene Exekutiventscheidungen ${ }^{33}$. Die Notwendigkeit, personelle Alternativen außerhalb des PRI zu suchen, wird dem unkritischen Bürger auf diese Weise abgenommen. Die Hoffnung auf einen anderen Präsidenten ist die auf einen Nachfolger und dessen Nachfolger. Die Herrschaft des PRI ist bereits psychologisch derart perpetuiert.

Gerhard Scheffler

\footnotetext{
25 Echeverría mußte, wie üblich, als Botschafter die Innenpolitik meiden. Sein Zugriff auf das Sexenium 1976-1982 wargescheitert, weder maximato noch auch nur mínimato.

26 Smith, S. 299 ff. Darunter $71 \%$ Absolventen der UNAM, Smith, S. 306

27 In der Inaugurationsrede vom 1. 12. 1976 als Forderung aufgestellt, Comercio Exterior 1976, S. 1459-1466, und seither ständig wiederholt.

28 Unter López P. ist auch der Anteil der Frauen in höherrangigen Positionen angestiegen, Smith, S. 307. 1979 wurde erstmals eine Frau, Griselda Alvarez, vormalige Senatorin, zur Gouverneurin in Colima gewählt. Parallel dazu hat López P. 1980 mit Rosa Luz Alegria als Touristikminister eine Frau in das Kabinett genommen.

29 Mols, in: Mols-Tobler, Mexiko. Die institutionalisierte Revolution, Köln-Wien, 1976, S. 171 (180).

30 Smith, S. $281 / 2$.

31 Die Deputiertenwahl vom 1. 7. 1979 brachte dem PRI 72,8 \% der gültigen Stimmen und damit 296 von 400 Sitzen in der Kammer, Comercio Exterior 1979, S. $733 \mathrm{ff}$

32 Diario de México v. 20. 3. 1980, S. 1 und 6.

33 Maza, in: Proceso v. 11. 1. 1977, S. 40 f
} 